\title{
COMBINED ORGANIC THERMAL AND ELECTROSTATIC OMNIDIRECTIONAL CILIARY MICROACTUATOR ARRAY FOR OBJECT POSITIONING AND INSPECTION
}

\author{
John W. Suh, Steven F. Glander, Robert B. Darling*, \\ Christopher W. Storment and Gregory T. A. Kovacs \\ Stanford University, Department of Electrical Engineering \\ Room CISX 202, M/C 4075, Stanford, CA 94305 \\ *Department of Electrical Engineering, Box 352500 \\ University of Washington, Seattle, WA 98185
}

\begin{abstract}
An organic thin film ciliary microactuator array using independent thermal and electrostatic actuation is described. A polyimide thermal bimorph structure provides for large angle deflection with high load capacity. Electrostatic plates provide a low power hold-down, capacitive sensing and feedback control capability. Integrating four orthogonally oriented actuators into a unit cell and replicating this cell into an array allows for precise movement of small objects in arbitrary directions. The array has immediate applications for precision positioning, alignment, inspection, and assembly of small parts, such as IC dice, with micron scale resolution.
\end{abstract}

\section{INTRODUCTION}

Several groups have described efforts to apply micromachined actuators to component positioning, inspection, and assembly [1-6]. These applications often require considerable excess force and large movement of the actuator. Despite the large number of papers on micromachined actuators, only a few have discussed the fabrication of microactuator arrays which can move more than their own weight.

The limitations of candidate actuation schemes can be lessened by combining two or more. Our approach is to use thermal actuation for the initial large-angle movement of each actuator and then transition to electrostatic actuation for a lowpower hold-down mode. While thermal bimorph actuators are well known, it appears that the only previous attempt to incorporate electrostatic actuation in an organic thermal actuator was that of Lin, et al. [7]. Their approach was to apply an electrostatic pull-down potential to an electrically noninsulated $\mathrm{Au}$ resistive heater loop of a thermal bimorph actuator. The devices presented herein contain fully independent (and electrically insulated from the external environment) thermal and electrostatic actuators within each element, allowing for uncomplicated implementation of electrostatic actuation and/or sensing in combination with simultaneous thermal actuation. In this paper, the design, fabrication, and testing of such thermal/electrostatic actuators is presented.

\section{FABRICATION}

Surface micromachining techniques were used to create the actuators using polyimide as the primary structural material and aluminum as the sacrificial layer. The fabrication process was designed to be compatible with CMOS or BiCMOS circuits which could be pre-fabricated on a silicon substrate. The process consists of nine lithographic steps using eight masks. Process cross-sections are shown in Fig. 1.

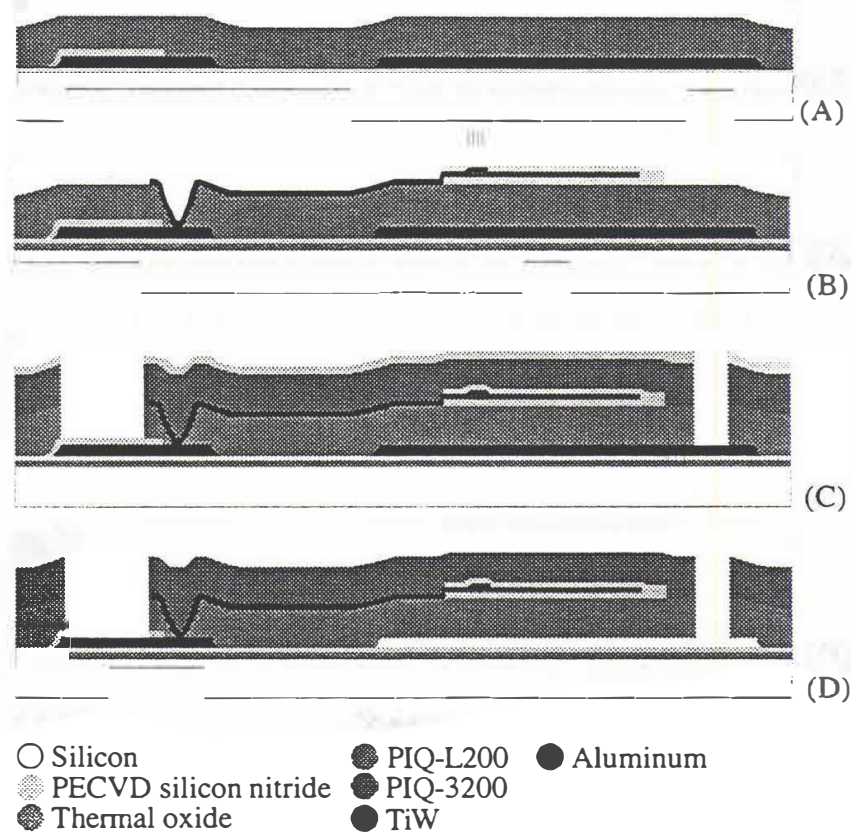

Figure 1. Process flow cross-sections for organic thermal/electrostatic actuator.

The steps up to and including the first polyimide layer are shown in Fig. 1(A). Starting with a thermally oxidized Si wafer, $0.25 \mu \mathrm{m}$ of $\mathrm{Al}$ was sputtered and pattemed to form alignment keys (Mask 1). Next, $0.5 \mu \mathrm{m}$ of LTO was deposited to encapsulate the $\mathrm{Al}$ alignment keys. Another $1.6 \mu \mathrm{m}$ of $\mathrm{Al}$ was sputtered and patterned to form sacrificial mesas, interconnects, and bond pads (Mask 2). Since this Al layer would later be exposed to a wet Al etch, a $0.5 \mu \mathrm{m}$ PECVD silicon nitride masking layer was deposited and patterned to cover the exposed bond pads (Mask 3). An adhesion promoter (Hitachi PI-coupler-3) was spun on and cured at $350^{\circ} \mathrm{C}$ for 30 minutes followed by spin on of the first (low thermal expansion coefficient) Hitachi PIQ-L200 polyimide. The polyimide was cured in a nitrogen purged oven using the a three step profile: 20 minutes at $105^{\circ} \mathrm{C}, 30$ minutes at $160^{\circ} \mathrm{C}, 60$ minutes at $350^{\circ} \mathrm{C}$. The final, cured lower polyimide thickness was $4 \mu \mathrm{m}$.

The following steps describe the processes performed on top of the first polyimide layer shown in Fig. l(B). A $150 \mathrm{~nm}$ PECVD silicon nitride layer was deposited and patterned as the etch mask for the $\mathrm{Al}$ interconnect vias (Mask 4). An isotropic oxygen plasma etch was used to etch vias in the first polyimide to the underlying $\mathrm{Al}$. The PECVD silicon nitride was repatterned to form the lower encapsulation layer for all subsequent metallizations (Mask 5). The silicon nitride also functions as an internal stiffener for the actuator. Next, $100 \mathrm{~nm}$ 
of 50:50 Ti:W was sputtered and patterned to form the resistive heaters (Mask 6). Electrical connections between the Ti:W and the first $\mathrm{Al}$ layer were made by sputtering and patterning 750 $\mathrm{nm}$ of $\mathrm{Al}$ (Mask 7). Another $150 \mathrm{~nm}$ of PECVD silicon nitride was deposited and patterned (reusing Mask 5) to complete metal encapsulation.

The remaining process steps are as follows. The second (high thermal expansion coefficient) Hitachi PIQ-3200 polyimide was spun on and cured in the same manner as the first. The final thickness was also $4 \mu \mathrm{m}$ after curing. Finally, $250 \mathrm{~nm}$ of PECVD silicon nitride was deposited and patterned (Mask 8) to form the actuator outlines, as shown in Fig. 1(C). The wafer was then diced. Afterwards, a RIE oxygen plasma was used to etch through the polyimide and stop either on the PECVD silicon nitride (over the bond pads) or on the sacrificial aluminum mesas. Individual chips were then placed in an aluminum wet etch to release the actuators from the substrate. A final plasma etch was done to remove the PECVD silicon nitride on the top polyimide layer as well as on the bond pads. The final profile is shown in Fig. 1(D).

Because the polyimides were cured at high temperature and because the upper polyimide layer has the larger thermal expansion coefficient, the actuators assume an upward, out-ofplane curl when the process is complete. Heating by the Ti:W resistors flattens them back towards the substrate. The natural tendency for the actuators to curl upwards assists the release process by reducing the chance of sticking and by allowing the etchant to more easily access the area underneath the actuator.

\section{ACTUATOR AND ARRAY DESIGN}

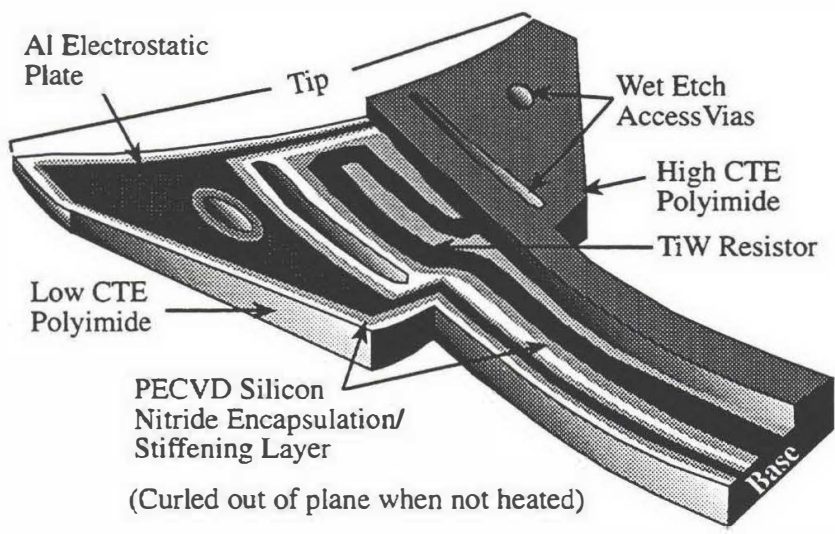

Figure 2. Illustration of a combined thermal and electrostatic ciliary microactuator.

Actuator elements. A single actuator is illustrated in Fig. 2, with half of the upper polyimide and PECVD nitride layers shown removed. The two wing shaped regions contain the electrostatic plates. These wings attach to the tip and midsection of the central, thermally actuated region.

The radius of curvature of a multi-layered structure as a result of thermal strain is

$$
R=\frac{h}{\varepsilon_{T}-\varepsilon_{B}}
$$

where $\varepsilon_{T}$ and $\varepsilon_{B}$ are the strains on the top and bottom layer of the actuator and $h$ is the combined thickness of the structure. Because the actuator has curvature along its length and width, the strain solutions used were those of a disk exhibiting axially symmetric strains $[8,9]$. Relative to its location before release from the substrate, the deflection of the actuator tip involves both vertical and horizontal displacements,

$$
\begin{gathered}
\delta_{V}=R\left(1-\cos \left(\frac{L}{R}\right)\right) \\
\delta_{H}=L-R \sin \left(\frac{L}{R}\right)
\end{gathered}
$$

where $\mathrm{L}=430 \mu \mathrm{m}$ is the length along the actuator's axis of symmetry when flat. Following release, the actuators assumed a radius of curvature of approximately $\mathrm{R}=706 \mu \mathrm{m}$, yielding displacements of $\delta_{v}=127 \mu \mathrm{m}$ and $\delta_{H}=26 \mu \mathrm{m}$

The lifting capacity of an actuator can be estimated as the force required to deflect the actuator's tip to the substrate,

$$
F_{L}=\frac{3}{L^{3}}(E I)_{\text {equiv }} \delta_{V}
$$

where $(E)_{\text {equiv }}$ is the equivalent flexural rigidity of the actuator $[10,11]$. Using the nominal parameters of the actuator layers listed in Table 1 , an actuator load capacity of $F_{L}=76$ $\mu \mathrm{N}$ is obtained.

Table 1. Layer parameters of fabricated actuator

\begin{tabular}{|c|c|c|c|c|c|c|}
\hline Layer \# & 1 & 2 & 3 & 3 & 5 & 6 \\
\hline Material & PIQ- & $\begin{array}{l}\text { PECVD } \\
\text { Silicon } \\
\text { Litride }\end{array}$ & Ti:W & Al & $\begin{array}{c}\text { PECVD } \\
\text { Nilicon } \\
\text { Nitride }\end{array}$ & $\begin{array}{c}\text { PIQ- } \\
3200\end{array}$ \\
\hline Thickness & $4 \mu \mathrm{m}$ & $150 \mathrm{~nm}$ & $100 \mathrm{~nm}$ & $780 \mathrm{~nm}$ & $150 \mathrm{~nm}$ & $4 \mu \mathrm{m}$ \\
\hline $\mathrm{E}(\mathrm{GPa})$ & 8.63 & 70 & N/A & 7 & 70 & 2.95 \\
\hline $\begin{array}{c}\mathrm{CTE} \\
\left(\mathrm{ppm} /{ }^{\circ} \mathrm{C}\right)\end{array}$ & 2.0 & 0.8 & N/A & 23.6 & 0.8 & 54 \\
\hline
\end{tabular}

The Ti:W heating elements were designed for a nominal resistance of $1500 \Omega$, dissipating $16.7 \mathrm{~mW}$ for an applied $5.0 \mathrm{~V}$. The wing electrodes were designed for an area of $24,400 \mu \mathrm{m}^{2}$, which yields a capacitance of $300 \mathrm{fF}$ for each actuator, when the actuator is fully flattened onto the substrate.

Chip layout. The omnidirectional ciliary array is composed of a two dimensional array of cells having four orthogonally oriented actuators each (Fig. 3). This $8 \times 8$ array of cells (each $1 \times 1 \mathrm{~mm}$ ) has a total active area of $0.64 \mathrm{~cm}^{2}$.

The four actuators of each cell are independently activated by 4 thermal and 4 electrostatic control lines. Within each column of 8 cells, the thermal elements for a given actuator orientation are wired in series, whereas the electrostatic elements for a given actuator orientation are wired in parallel. The eight columns are then wired in parallel for both the thermal and electrostatic elements. Each electrostatic control line (E1 - E4) applies a potential to the wing electrodes of all 64 actuators of a given orientation. Similarly, each thermal control line ( $T 1$ - T4) runs a current through the Ti:W heaters of all 64 actuators of a given orientation, such that the load resistance for the overall array is the same as that of a single element $(\approx 1500 \Omega$ ). A common ground bus provides a current return path for the thermal elements, and a substrate contact provides an opposing plate for the electrostatic elements. 

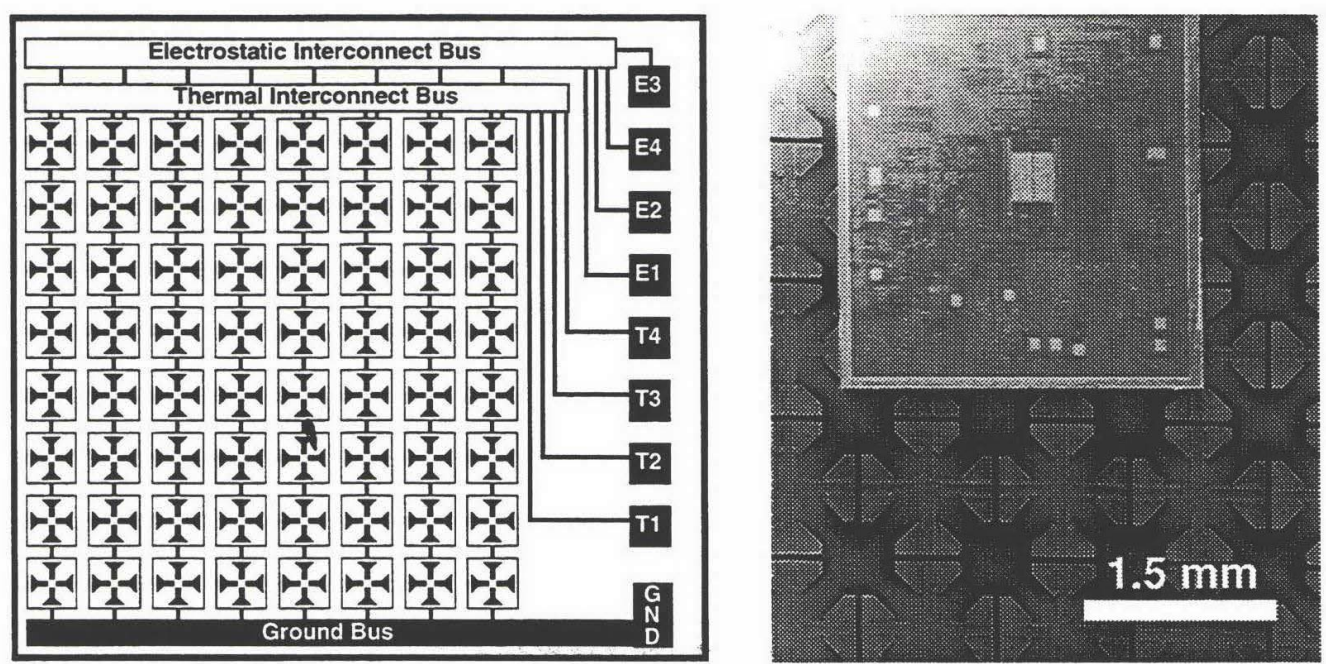

Figure 3. Schematic (not to scale) of the omnidirectional ciliary microactuator array chip (lefi). The SEM photograph on the right shows part of the array with an ADXL5O accelerometer (courtesy of Dr.R Payne, Analog Devices, Inc.)
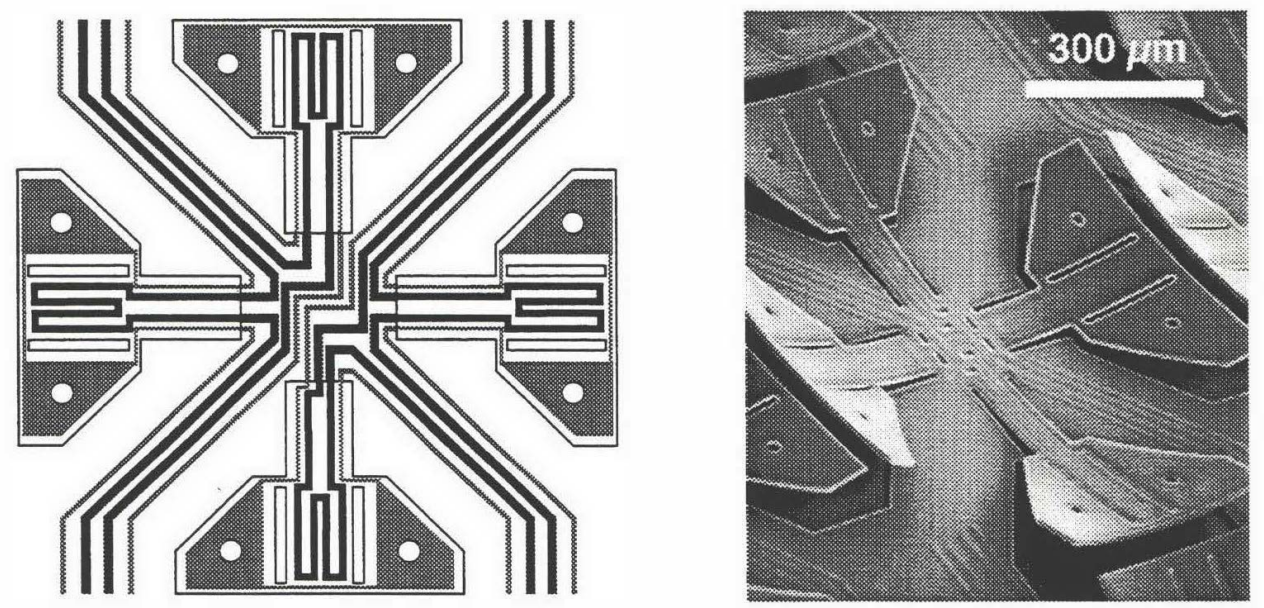

Figure 4. Illustration of a motion pixel (left). Four actuators in a common center configuration make up each pixel. Thicker, darker lines represent Al interconnects for the Ti:W resistors. Thinner, lighter lines represent Al interconnects for the electrostatic plates. The SEM photo on the right shows an oblique view of a motion pixel.

\section{EXPERIMENTAL RESULTS}

Lifting capacity. Lifting capacity tests were performed to determine how massive an object the ciliary array could convey. The array successfully moved objects with different masses at widely variable step rates which were independent of their mechanical resonances. The objects included a $3 \times 3 \mathrm{~mm}$ Si IC $(8.6 \mathrm{mg})$, a $1.0 \times 1.5 \times 1.5 \mathrm{~mm}$ glass block $(15 \mathrm{mg})$, a \# 4 40 stainless steel nut $(0.16 \mathrm{~g})$ and an 8 -pin plastic DIP package $(0.5 \mathrm{~g})$. The DIP package exerted nearly $69 \mu \mathrm{N} / \mathrm{mm}^{2}$ which is near the maximum theoretical lifting capacity of a single actuator $(76 \mu \mathrm{N})$ (even though there are four actuators in a motion pixel, at times only one actuator in a pixel supports an object).

Four phase gait and arbitrary motion direction. Lateral movement of an object across the array is accomplished by applying a coordinated gait to differently oriented actuators. Figure 5 illustrates the basic four phase gait required to move the supported object in a rightward direction. Initially, all actuators are un-deflected and contact the object. Next, the updown actuator pair and the left actuators are deflected downward to allow the object to rest only on the right actuators.
The basic gait is established by applying two square waves to the left-right actuator pair with a relative phase delay of one quarter period. Deflecting the right actuators downward lowers the object by $\delta_{V}$ and translates it to the left by $\delta_{H}$. Deenergizing the left actuators raises the object by $\delta_{V}$ and translates it to the right by another $\delta_{H}$ increment. De-energizing the remaining actuators completes the cycle. The size of the horizontal and vertical displacement steps $\delta_{H}$ and $\delta_{V}$ are controlled by the voltage applied to the thermal actuators.

The above sequence, with appropriate rotation, describes object movement in each of the four primary (up, down, left, and right) directions. By actuating adjacent pairs with the same drive signals, motion along the diagonals can also be directly produced. Motion in any arbitrary direction was produced by a vector-to-raster algorithm based on that of Foley and Van Dam [13]. A PC-controlled system for producing the correct gait combinations and driving the array was developed and tested. The system allows for speed control via the drive pulse frequency and arbitrary directional control via keyboard or mouse. This system was used for performing the tests detailed below. 


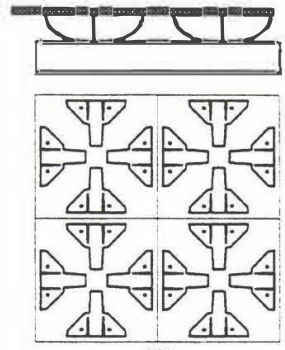

(1)

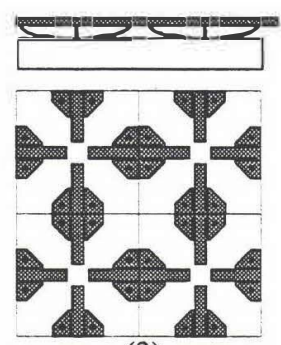

(3)

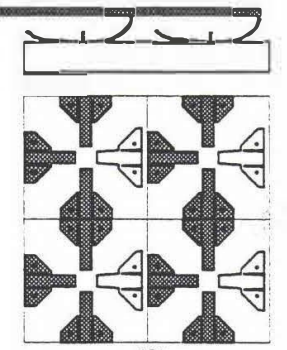

(2)

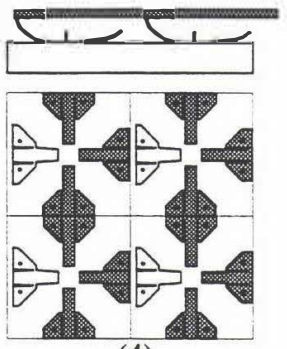

(4)
Figure 5. Side and plan views of four phase gait. Side views show object movement over actuators. In the plan view, dark colored actuators are thermally deflected downward.

Four-phase, linear gaits and step size. The actuators were operated at widely variable step rates. The effect this had on the linear movement of the $3 \times 3 \mathrm{~mm}$ silicon IC was examined and the results are summarized in Fig. 6 below. When the time taken for all four phases (gait period) illustrated in Fig. 5 above is below $80 \mathrm{msec}$, the distance the object moves in one gait or distance per step varies nearly linear with gait period. Above $\approx 120 \mathrm{msec}$ the distance the object moved per gait plateaued at an average of $9 \mu \mathrm{m}$ per cycle. This behavior is related to the thermal time constant of the actuator. Longer gait periods allow the actuator to approach its steady state temperature. When the gait period is shorter, the actuator does not deflect as much and so the resulting distance per cycle is less.

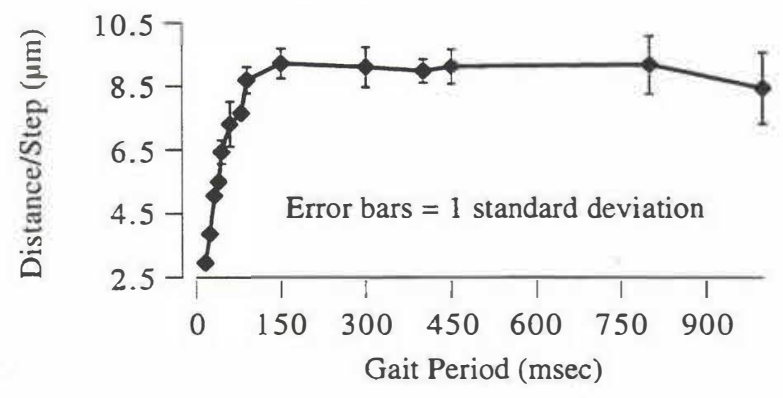

Figure 6. Plot of average distance a $3 \times 3 \mathrm{~mm}$ silicon IC moved per gait as gait period is increased from $16 \mathrm{msec}$ to $1 \mathrm{sec}$. The error bars for periods $\leq 40 \mathrm{msec}$ are smaller than the dots representing their averages.

Rotation, alignment, and centering. A set of four ciliary microactuator arrays was also packaged and tested. Takeshima and Fujita [12] and Böhringer, et al.[1] have discussed the potential for a distributed micro-motion system to manipulate objects using sensorless methods. They describe how distributed micro motion systems can perform such tasks as object rotation, alignment to a given axis, and centering objects at the center of an array. With this four chip set, these manipulation tasks have been demonstrated and are shown in Fig. 7.

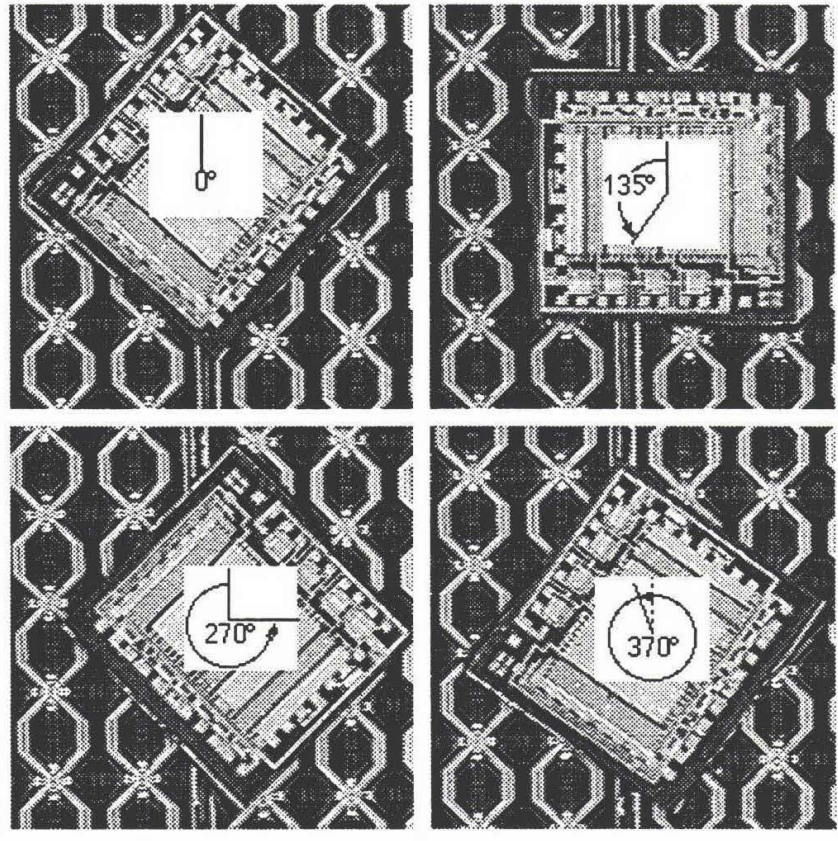

(A) Object rotation through $370^{\circ}$

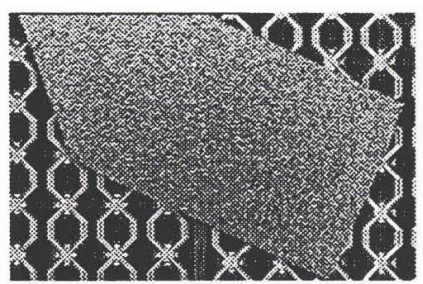

(1)

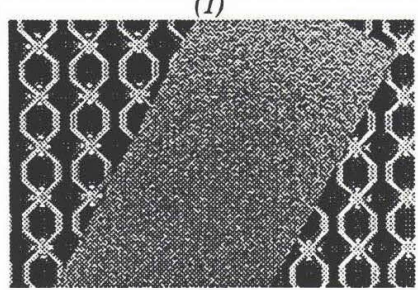

(3)

(B) Object's longitudinal axis aligned to vertical axis of microactuator array.

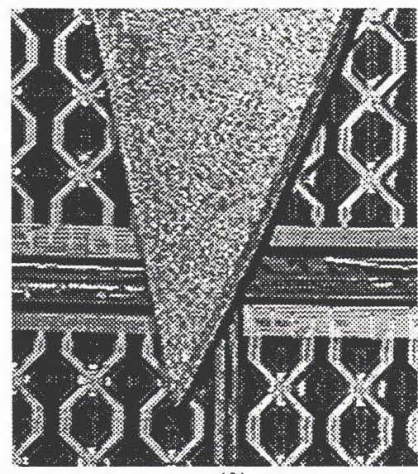

(l)

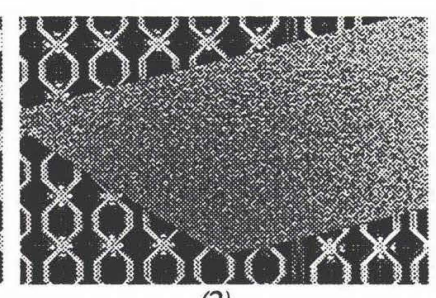

(2)

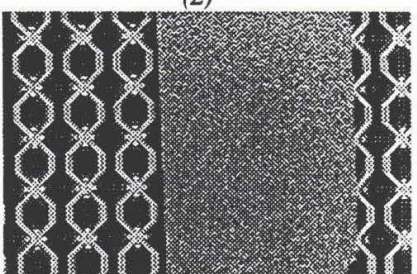

(4)
(C) Object positioned in center of four ciliary microactuator array chips.

Figure 7. Video frames of four omnidirectional ciliary microactuator arrays performing object rotation, alignment, and centering. 
Electrostatic Actuation. As thermal actuators are well known to have high power requirements, a low-power electrostatic hold-down mode becomes very attractive. The Al electrostatic plates are embedded symmetrically within the layers of nitride and polyimide of each actuator, and can be used to hold the actuator down on the substrate, or to hold an object onto a pair of actuators by the application of a voltage between the pair.

The capacitance of an actuator relative to the $\mathrm{Si}$ substrate involves a stack of several dielectric layers. When an actuator is flattened against the substrate (zero air gap), the layers include $0.15 \mu \mathrm{m}$ of PECVD nitride $\left(\varepsilon_{\mathrm{r}}=7.5\right), 4 \mu \mathrm{m}$ of polyimide $\left(\varepsilon_{\mathrm{r}}=3.4\right), 0.5 \mu \mathrm{m}$ of LTO $\left(\varepsilon_{\mathrm{r}}=7.5\right)$, and $0.5 \mu \mathrm{m}$ of thermal oxide $\left(\varepsilon_{\mathrm{r}}=7.5\right)$. The capacitance of this stack is

$$
C=\frac{A}{\frac{d_{1}}{\varepsilon_{1}}+\frac{d_{2}}{\varepsilon_{2}}+\frac{d_{3}}{\varepsilon_{3}}+\frac{d_{4}}{\varepsilon_{4}}}
$$

If fringing fields are neglected, the hold-down voltage is

$$
V \approx \sqrt{\frac{2 F_{L} \varepsilon_{0}}{C}\left(\frac{d_{1}}{\varepsilon_{1}}+\frac{d_{2}}{\varepsilon_{2}}+\frac{d_{3}}{\varepsilon_{3}}+\frac{d_{4}}{\varepsilon_{4}}\right)}
$$

where $F_{L}$ is the force required to flatten the actuator and $\varepsilon_{0}$ is the permittivity of the air gap. The above parameters predict a hold-down voltage of $27 \mathrm{~V}$.

Figure 8 illustrates the deflection characteristics of the actuators to both thermal and electrostatic actuation. When the actuators are in their full upward position, their capacitance to the substrate is very low and $500 \mathrm{~V}$ of applied bias only produces a downward deflection of $15 \mu \mathrm{m}$. In comparison, 16.7 $\mathrm{mW}$ applied to the thermal elements produces a downward tip deflection of nearly $30 \mu \mathrm{m}$. Predeflecting the actuators downward with the thermal elements greatly reduces the electrostatic voltage required to pull down and hold the actuators. Pull-in voltages of 300 to $500 \mathrm{~V}$ were observed for 16 to $20 \mathrm{~mW}$ of initial thermal power per actuator. Once the actuators were snapped down, holding voltages of only $70 \mathrm{~V}$ were required, both with and without simultaneous thermal actuation. The observed $70 \mathrm{~V}$ hold-down voltage is larger than the predicted $27 \mathrm{~V}$ value, probably because the Si substrate provides a less than perfect ground plane.

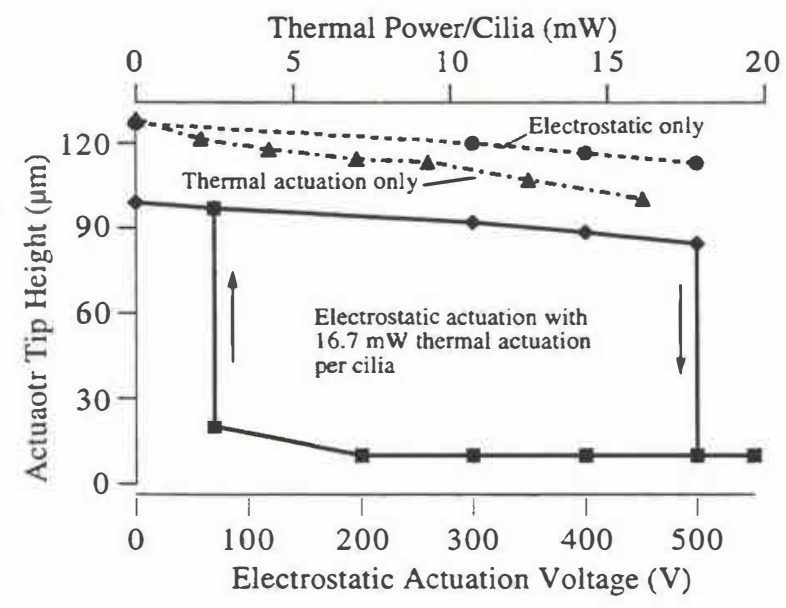

Figure 8. Deflection characteristics for electrostatic only, for thermal only, and for combined thermal (at $16.7 \mathrm{~mW}$ per cilia) and electrostatic actuation.

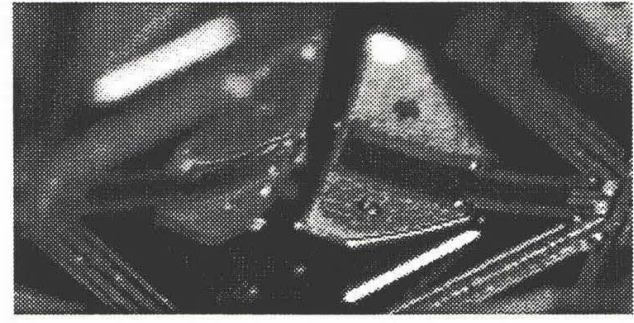

(A)
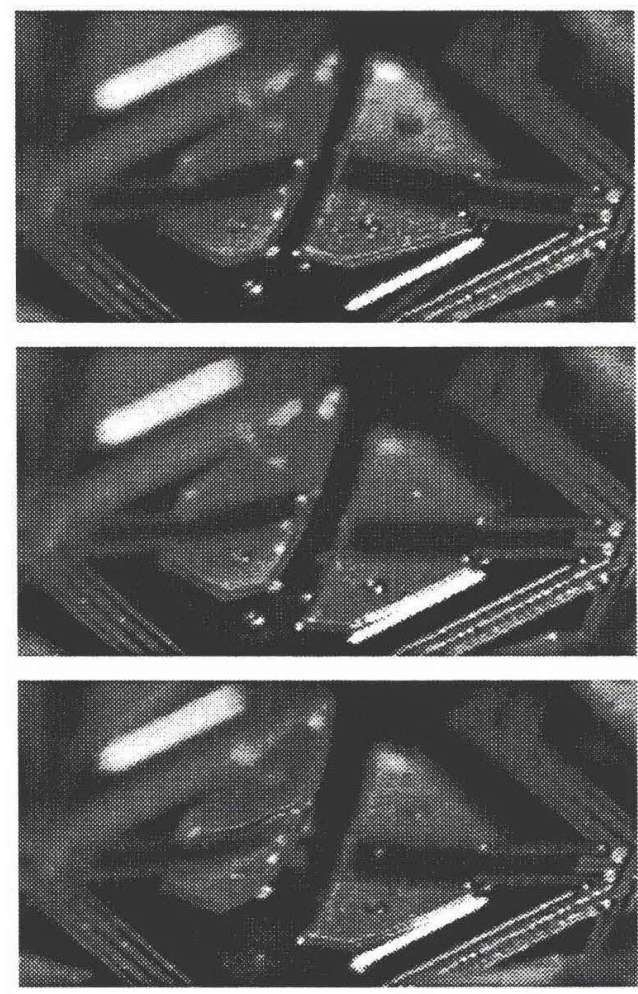

(D)

Figure 9. Video frames of actuators during combined mode thermal and electrostatic actuation. A) Unpowered, initially curled actuators. B) $16.7 \mathrm{~mW}$ of applied power to both actuators. C) Right actuator with $500 \mathrm{~V}$ applied to electrostatic plates. D) Left actuator unpowered, returns to same position as in (A).

By applying a differential voltage between neighboring actuators, conductive objects can be drawn down onto the actuators. This causes the actuator to flatten out against the object, drawing both the actuator and the object closer to the substrate, as shown in Fig. 10.

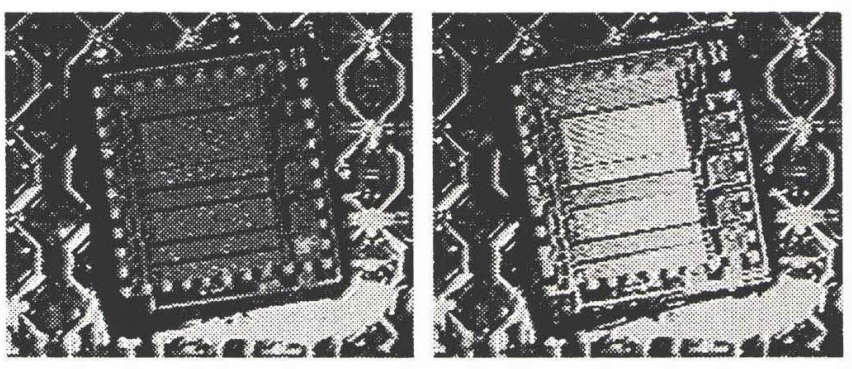

Figure 10. Optical video frames showing electrostatic hold down of an IC die in deionized water. Tilting of the chip as it is drawn down on top of the actuators is seen as a change in light reflected from its surface. 
Capacitive Sensing of Objects. If the array were constructed to allow independent addressing of each actuator, electrostatic sensing could be used for object weighing and/or location. The feasibility of this concept was examined. A circuit sensitive to capacitive changes resulting from changes in actuator position was built. A simple, balanced race condition circuit was designed using 4000 series CMOS gates which produces glitch pulses whose width is proportional to the nodal capacitance. Low pass filtering of this pulse train produces a DC voltage proportional to the capacitance. The response of this circuit was recorded while manually loading and then unloading all the actuators. The results are summarized in Fig. 11. below. The results indicate that sensing object weight/location by capacitive sensing is possible.

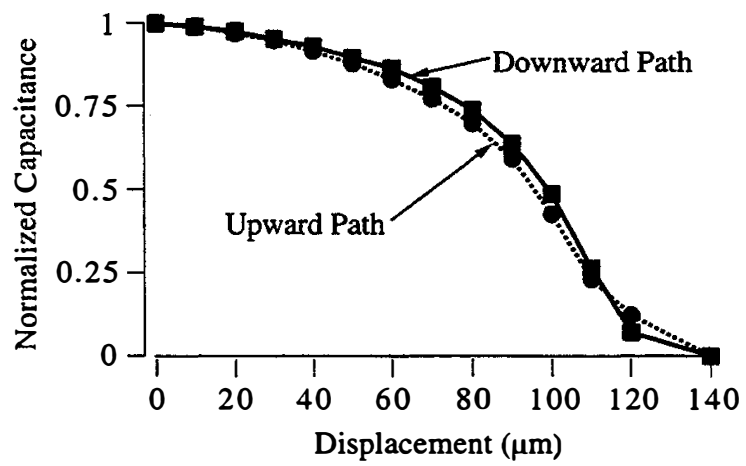

Figure 11. Vertical deflection of actuators (produced manually through micrometer) and change in voltage (proportional to capacitance). The actuator's tip is undeflected when displacement is set to zero.

\section{CONCLUSIONS AND OUTLOOK}

A ciliary microactuator array using independent thermal and electrostatic actuation has been described. The fabrication is based upon polyimide thermal bimorphs with aluminum sacrificial release layers and is compatible with prefabricated CMOS circuits. The combination of thermal and electrostatic actuation provides the simultaneous features of a high lifting capacity, large angle deflection mode with a low power holddown mode. Capacitive sensing of the actuator position can be used for position feedback control and object sensing. The $8 \times 8$ array has demonstrated applicability for precision positioning, alignment, and inspection of IC dice with micron resolution.

Further system demonstrations and enhancements are planned. Design efforts are underway to lower electrostatic actuation voltages to practical levels $(\leq 100 \mathrm{~V})$ by redesign of the shape and position of the electrostatic plates as well as layer thicknesses. In addition, capacitive sensing for feedback control and object measurement is being investigated. A ciliary microactuator array with pre-fabricated CMOS circuits, which would enable control of individual cilia, is also planned.

\section{ACKNOWLEDGMENTS}

This research was supported by grant no. N00014-92-J1940 from the Advanced Projects Research Agency (ARPA), managed by the Office of Naval Research. Special thanks are extended to Drs. Ken Gabriel and Teresa McMullen for supporting this effort. The authors give thanks to Karl Böhringer for his much appreciated contributions during testing. Thanks also go to Ken Honer and Dario Falquier.

\section{REFERENCES}

1. K.-F. Böhringer, B. R. Donald, R. Mihailovich, N. C. MacDonald, "A Theory of Manipulation and Control for Microfabricated Actuator Arrays," in Proc. IEEE Micro Electro Mechanical Systems, MEMS '94, pp. 102-107, 1994.

2. C. Liu, T. Tsao, Y.-C. Tai, W. Liu, P. Will, C.-M. Ho, "A Micromachined Permalloy Magnetic Actuator Array for Micro Robotics Assembly Systems," in Transducers - Digest 8th Int. Conf. on Solid-State Sensors and Actuators/Eurosensors IX, vol. 1, pp. 328-331, Stockholm, Sweden, June 1995.

3. N. Takeshima and H. Fujita, "Polyimide Bimorph Actuators for a Ciliary Motion System." in ASME WAM, Symp. Micromech. Sensors, Actuators, and Systems, DSC-vol. 32, pp. 203-209, 1991.

4. K. S. J. Pister, R. Fearing, and R. Howe, "A Planar Air Levitated Electrostatic Actuator System." in Proc. IEEE Workshop on Micro Electro Mechanical Systems, pp. 67-71, Napa Valley, CA, February 1990.

5. S. Konishi and H. Fujita, "A Conveyance System Using Air Flow Based on the Concept of Distributed Micro Motion Systems," in Transducers-Digest 7th Int. Conf. on Solid-State Sensors and Actuators, pp. 28-31, Pacifico, Yokohama, Japan, June 1993.

6. T. Furuhata, T. Hirano, and H. Fujita, "Array-Driven Ultrasonic Microactuators," in Transducers - Dig. Int. Conf. on Solid-State Sensors and Actuators, pp. 1056-1059, Montreux, France, June 1991.

7. G. Lin, C.-J. Kim, S. Konishi, and H. Fujita, "Design, Fabrication, and Testing of a C-Shape Actuator," in Transducers - Digest 8th Int. Conf. on Solid-State Sensors and Actuators/Eurosensors IX, pp. 416-419, 1995.

8. P. M. Hall, F. L. Howland, Y. S. Kim, and L. H. Herring, "Strains in Aluminum-Adhesive-Ceramic Tri Layers," ASME $J$. Electronic Packaging, vol. 112, pp. 288-302, 1990.

9. P. M. Hall, F. L. Howland, Y. S. Kim, and L. H. Herring, "Errata", ASME J. Electronic Packaging, vol. 113, pp. 26, 1991.

10. W.-H. Chu, M. Mehregany, and R. L. Mullen, "Analysis of Tip Deflection and Force of a Bimetallic Cantilever Microactuator," J. Micromech. Microeng., vol. 3, pp. 4-7, 1993. 11. J. Söderkvist, "Similarities Between Piezoelectric, Thermal and Other Internal Means of Exciting Vibrations," $J$. Micromech. Microeng., vol. 3, pp. 24-31, 1993.

12. N. Takeshima and H. Fujita, "Design and Control of Systems with Microactuator Arrays," in Proc. IEEE Workshop on Advanced Motion Control, pp. 219-232, Yokohama, Japan, March 1993.

13. J. D. Foley and A. Van Dam, Computer Graphics: Principles and Practice, 2nd Edition, 1996. 